\title{
Hemi-retinal attention under conditions of color rivalry
}

\author{
JAMES J. BARRELL AND THEODORE PARKS1 \\ UNIVERSITY OF CALIFORNIA, DAVIS
}

Human Ss viewed stimulus arrays of color patches such that two instances of color rivalry were simultaneously present. That is, two patches of different color were present, one each in each of the left halves of the two ocular fields, and at the same time, rival patches were also present in the two right halves. As would be expected, binocular percepts often corresponded to predominance of one or the other of the two eyes. On other occasions, however, Ss reported percepts which indicated simultaneous predominance of the nasal halves or, more often, of the temporal halves of the two retinas.

Corballis (1964) reported interesting intraocular differences in the ability of human Ss to recognize tachistoscopic stimuli. When letters were presented to each half of the visual field of each eye, accuracy of recognition was greater for letters in the left (than right) half of the field of the left eye. In addition, accuracy was greater in the right (than left) half of the right-eye field. Thus, as measured by letter recognition, performance based on the nasal half of each retina was superior to that of the temporal hemiretina of the same eye. ${ }^{2}$

Superior performance of the nasal hemiretinas, as reported by Corballis, might reflect a slight, but constant, advantage in acuity for those hemiretinas. Alternatively, Corballis' results are not inconsistent with the hypothesis that, on various occasions during the course of his experiments, several forms of relatively extreme hemiretinal advantage occurred. That is, of the four hemiretinas, strong predominance of the two hemiretinas of the left eye, or of the right eye, or of the two temporal hemiretinas, as well as of the nasal hemiretinas, might have been present for brief intervals. Under this hypothesis, the overall tendency toward superior recognition of information presented to the nasal hemiretinas would be taken to indicate that attention to those hemiretinas was simply more common than predominance of the temporal hemiretinas.

Elaborating this latter hypothesis, predominance of the nasal (or of the temporal) hemiretinas may occur to the same degree that attention to stimulation of the left or right eye occurs under conditions of binocular rivalry (see, for example, Woodworth \& Schlosberg, 1962, pp. 399-401). Thus, voluntary or involuntary attention to stimulation falling on one of the two eyes would not be the only means whereby the visual system tends to form a single, whole field-of-view from conflicting stimulation falling on the four hemiretinas.

In the present study, the resolution of color rivalry, rather than letter recognition, was employed as a measure of the contribution of each of the four hemiretinas. For example, a color patch was presented in the left half of the left-eye field which contrasted with a patch in the corresponding part of the left-half of the right visual field (see Fig. 1). The color, if either, which was reported as appearing in the left side of S's binocular field at any moment was taken as an indication of which, if either, of the two hemiretinas involved predominated at that time. Simultaneously, conflicting colors were also present in the right halves of the two visual fields. Again, the color, if either, which was reported as appearing to the right would indicate momentary dominance of either the temporal hemiretina of the left eye or of the nasal hemiretina of the right eye. Since reports on the left and right halves of the visual field were taken simultaneously, the occurrence of any of the four forms of dominance mentioned above could be detected.

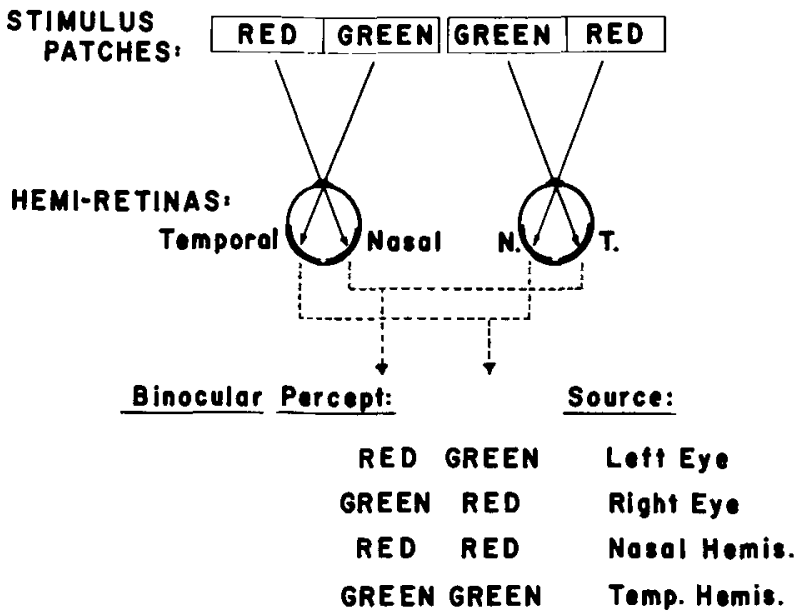

Fig. 1. A diagramatic representation of a typical stimulus condition used in the present study. The upper portion shows one of the four arrangements of color patches (the gap between the two patches presented to each eye has been omitted for clarity). The interpretation of each form of binocular percept that may be reported (bottom portion of figure) depends upon the particular arrangement of patches that is presented.

\section{Subjects}

\section{METHOD}

A total of 20 naive university students participated in the present study. However, preliminary difficulties with binocular fusion decreased the number who completed the experiment to 14 .

\section{Apparatus and Materials}

Stimuli were presented in a viewing box which was similar in general form and size to that employed by Corballis. That is, the stimuli were located at one end of a $42 \times 6 \% \times 1 / 4$ in. (inside dimensions) wooden tunnel painted flat black. A partition through the center of this tunnel divided into two $42 \times 3 \frac{1}{4} \times 3 \frac{1}{4}$ in. tunnels, each terminating in a square viewing area of the latter dimensions. A cushioned mask was located at the opposite end of the box such that, in proper position, each eye viewed one and only one of the two viewing surfaces.

The stimuli, themselves, were mounted on semiopaque white card stock (120 wt) which was illuminated from behind by two $25-\mathrm{W}$ incandescent bulbs (G.E. 25T6-1/2), one centered behind each viewing area at a distance of $2 \mathrm{in}$. The resulting surfaces had a luminance of approximately $30 \mathrm{ft}-\mathrm{L}$.

Stimuli consisted of $1 / 2 \times 9 / 16$ in. rectangles of green or red celluloid. On any trial, two such stimulus patches were presented on each viewing surface, alligned horizontally at a separation of $7 / 8 \mathrm{in}$. and centered vertically and equally distant from the horizontal center of the area. If a given eye were fixated on the area between the two patches presented to that eye, one of those patches would have stimulated that nasal hemiretina and the other that temporal hemiretina.

\section{Procedure and Design}

Each $S$ participated in four experimental sessions, each session being on a separate day and each involving a different pattern of stimulus patches for that entire session. The 
arrangement of patches employed during any session was: red-green-green-red (as in Fig. 1), or green-red-ted-green, or red-red-green-green, or green-green-red-red (reading from left to right across the viewing fields). The order of these conditions was random for each $S$.

On each trial within a session, Ss were asked to fuse the two patches of the left eye with the two of the right. It should be noted that contrary to Corballis' findings (1964, p. 39), most Ss reported some difficulty in achieving such fusion and, in fact, six were unable to do so and, therefore, were unable to complete the experimental procedure.

Once fusion was accomplished, Ss were required to observe which, if either, color was seen uniformly in the left-hand rectangle, and simultaneously which, if either, was seen in the right-hand rectangle. Specifically, on each trial Ss were instructed to press a key whenever, and as long as, a particular, specified combination of left and right colors was simultaneously present in the binocular percept. For example, on a particular trial they might have been asked to press a key whenever both the left rectangle was uniformly red and the right was uniformly green. The key activated a Standard electric clock and total key depression on each trial was, thereby, recorded. As explained above, such responses may be interpreted as reflecting predominance of the left eye, the right eye, the nasal hemiretinas, or the temporal hemiretinas depending upon the percept that was being reported and on the arrangement of stimulus patches that was present during that session.

Each session consisted of four 60 -sec trials. On these four trials, Ss were required to report percepts of green-green, red-red, red-green, and green-red on the first through fourth trials, respectively. Since each of these percepts reflected a different form of hemiretinal attention under each of the four patterns of stimulus patches that was employed and since they were always reported in this order, any effect of type of percept or of time within a session is balanced across the four forms of hemiretinal attention when the experiment is taken as a whole.

\section{RESULTS}

Every $S$ reported occasional percepts which could be interpreted as reflecting instances of the predominance of the nasal hemiretinas. That is, for trials on which the percept to be reported corresponded to the stimulation that was falling on the nasal hemiretinas, Ss reported the presence of that percept for a mean of 8.3 of each $60-\mathrm{sec}$ trial.

However, some instances of predominance of the temporal hemiretinas were also reflected in each S's reports. In fact, this form of hemiretinal attention was reported more frequently than nasal predominance by 13 of the 14 Ss. Across Ss, it occurred for a mean of $11.5 \mathrm{sec}$ per $60-\mathrm{sec}$ trial $(\mathrm{F}=27.71$, $\mathrm{df}=1 / 13, \mathrm{p}<.01)$.

Finally, as might be expected, instances of both left-eye and right-eye predominance were also reported by all Ss. Specifically, mean total durations per trial of 10.4 and $16.3 \mathrm{sec}$ were reported for the left and right eyes, respectively. The apparent prevalence of these forms of attention over the intraocular forms was not reliable $(\mathrm{F}=3.83, \mathrm{df}=1 / 13)$.

It will be noted that the mean durations for each of the four forms of predominance do not sum to $60 \mathrm{sec}$. This finding is consistent with spontaneous verbal reports by Ss of percepts involving at least two colors within either or both parts of the perceived stimulus pattern.

\section{DISCUSSION}

The present findings extend the Corballis effect to include the resolution of color rivalry. That is, since instances of predominance of the nasal hemiretinas were found under the present conditions, it is apparent that attention to those hemiretinas may be so extreme as to preempt simultaneous color stimulation of the temporal hemiretinas. Furthermore, however, evidence was also found for comparable attention to both of the temporal hemiretinas. It would appear, therefore, that the visual system is capable of resolving binocular conflict by suppressing, at least momentarily, information presented to either of each pair of hemiretinas. Thus, the overall superiority of the nasal hemiretinas reported by Corballis might have reflected a relative prevalence of nasal over temporal predominance under his experimental conditions.

The conclusion that these perceptual effects may be attributed to uniform action of each hemiretina as a whole must be defended in some detail. That is, within each of a given pair of conflicting patches, rivalry may have occurred and been resolved in units that were smaller than the patches themselves. Secondly, even if each patch tended to predominate (or be predominated by) its rival patch as a whole, it might still have been the case that other parts of the hemiretina that lay outside the area stimulated by the patch did not share in that predomination.

With respect to the former objection, in a recent experiment Whittle, Bloor, and Pocock (1968, Experiment 3) presented two simple figural stimuli to the nasal hemiretina of the left eye and, simultaneously, two conflicting stimuli to and around corresponding points on the temporal hemiretina of the right eye. Although they found a tendency for their Ss to report percepts involving either both of the stimuli of one hemiretina or both stimuli presented to the other hemiretina, their Ss also gave frequent reports of percepts involving, simultaneously, one of the stimuli from the temporal hemiretina and one of the stimuli from the nasal hemiretina. Thus, within each hemiretina one instance of rivalry was resolved in favor of that hemiretina and one in favor of the conflicting hemiretina. Extending this phenomenon to relatively large, conflicting color patches, each patch might be considered to have been composed of an unspecified, large number of independent points, each of which had an even probability of predominating over the corresponding point of the contralateral patch. In that event, however, the probability that all such independent points on one patch would simultaneously predominate all points of the opposite patch would have been extremely small (how small depending, of course, on how many such points existed within each patch). Apparently, then, the tendencies toward predomination of the various parts of each patch were not independent but were somehow synchronized.

It may be, however, that such a coordinated effect within each patch simply depends upon all such points being parts of a simple, continuous stimulus pattern (Whittle et al, p. 186). But that phenomenon, taken alone, could not explain why predominance of the temporal patches was reliably more frequent than that of the nasal patches in the present study. It is clear that the present method cannot demonstrate that the two temporal patches acted in unison as opposed to the possibility that the overall percepts of "temporal predominance" simply reflected the simultaneous resolutions of two independent rivalries (both being in favor of the temporal patch involved). Nevertheless, it is also clear that if the two rivalries are independent then it must be that in both cases the temporal patch was favored (thus making a temporal percept more likely than a nasal percept since the former is the combination of two relatively likely events). Parsimony would, in turn, suggest that this tendency is a property of each of those hemiretinas. In this sense, it would appear that tendencies toward momentary hemiretinal dominance are akin to tendencies toward eye dominance.

\section{REFERENCES}

CORBALLIS, M. C. Binocular interactions in letter recognition. Australian Journal of Psychology, 1964, 16, 38-47. 
RUCH, T. C., PATTON, H. D., WOODBURY, J. W., \& TOWE, A. Neurophysiology. Philadelphia: Saunders, 1961.

WHITTLE, P., BLOOR, D. C., \& POCOCK, S. Some experiments on figural effects in binocular rivalry. Perception \& Psychophysics, 1968, 4, 183-188.

WOODWORTH, R. S., \& SCHLOSBERG, H. Experimental psychology. New York: Holt, Rinehart, \& Winston, 1962.

\section{NOTES}

1. Address: Department of Psychology, University of California, Davis, Calif. 95616.
2. A vertical line drawn through the center of the fovea divides the retina into two halves called "hemiretinas." Such a division of the retina is not arbitrary since it corresponds very closely to a difference in afferent pathways (see, for example, Ruch, Patton, Woodbury, \& Towe, 1961, p. 455).

(Accepted for publication September 24, 1968.) 